\title{
A large-scale collection of giant congenital melanocytic nevi: Clinical and histopathological characteristics
}

\author{
MIN WU*, QINGXIONG YU*, BOWEN GAO, LINGLING SHENG, QINGFENG LI and FENG XIE \\ Department of Plastic and Reconstructive Surgery, Shanghai Ninth People's Hospital, Shanghai Jiao Tong University, \\ School of Medicine, Shanghai 200011, P.R. China
}

Received August 31, 2017; Accepted January 3, 2018

DOI: $10.3892 /$ etm.2019.8198

\begin{abstract}
Giant congenital melanocytic nevi (GCMN) place a heavy psychological burden on patients due to their poor cosmetic appearance. The histopathological characteristics of GCMN have remained largely elusive. The present study investigated the histopathological characteristics of GCMN in association with their clinical appearance. A total of 98 patients diagnosed with GCMN were included in the present study and their clinical features were collected from their records. Lesion specimens were obtained and stained for histopathological analysis. Regarding the microscopic appearance of GCMN, nevi cells in the whole dermis exhibited different patterns than those in healthy tissues. Most GCMN cases featured a sub-epidermal non-involvement zone, which implies an early occurrence in embryo development. Darker nevi exhibited a higher density of infiltrated nevi cells and more pigment deposition; this appears to induce a poor skin texture. Chemical peeling and laser therapy only partly removes pigment particles and nevi cells in the upper portion of the dermis. The clinical features of GCMN are associated with the histopathological characteristics, and non-surgical therapy cannot remove the nevus cells in the deep dermis.
\end{abstract}

\section{Introduction}

Congenital melanocytic nevi (CMN) are present at birth or arise within the first few weeks of life. Their size has been used as the principal criterion for classification. Kopf et al (1)

Correspondence to: Professor Qingfeng Li or Professor Feng Xie, Department of Plastic and Reconstructive Surgery, Shanghai Ninth People's Hospital, Shanghai Jiao Tong University, School of Medicine, 639 Zhizaoju Road, Huangpu, Shanghai 200011, P.R. China

E-mail: dr.liqingfeng@yahoo.com

E-mail:dr.xiefeng@yahoo.com

*Contributed equally

Key words: clinical, histopathological, congenital, giant nevi, non-surgical therapy proposed the classification system currently in use according to which CMN are classified into 3 groups: Small, medium and large/giant. Small CMN are $<1.5 \mathrm{~cm}$; medium $\mathrm{CMN}$ measure $1.5-19.9 \mathrm{~cm}$ and large or giant $\mathrm{CMN}$ are $\geq 20 \mathrm{~cm}$ in projected adult size (PAS).

Giant congenital melanocytic nevi (GCMN) often present with an unsightly appearance that cannot be covered with normal dressing; thus, GCMN places a considerable psychological burden on the patients as well as their parents. The estimated prevalence of CMN is 1-6\% among all newborn infants (2), while GCMN (which are much rarer) are estimated to affect 1 in 20,000 newborns $(3,4)$.

It has been reported that the risk of melanoma in a patient with $\mathrm{CMN}$ increases with the size of the nevus $(5,6)$. In patients with a GCMN, the lifetime melanoma transformation risk is as high as $5-10 \%$, much higher than that for CMN and the common acquired melanocytic nevi (AMN) $(7,8)$. This higher malignant rate warrants increased attention of clinicians to GCMN; however, relatively few studies are dedicated exclusively to GCMN. Furthermore, in-depth studies on the histopathological features of GCMN are currently lacking. Studies suggest that CMN may have different genetic signatures; most cases of GCMN (97.4\%) have a mutation in the gene neuroblastoma RAS (9).

To date, the etiology and molecular mechanisms of GCMN have remained largely elusive and an enhanced knowledge in this field will facilitate the exploration of novel treatments. The present study aimed to determine the histopathological properties of GCMN in a series of 98 cases and to investigate how these are associated with the clinical presentation of this skin condition.

\section{Materials and methods}

Selection of cases. Patients diagnosed with GCMN and admitted to the Plastic Surgery Department of Shanghai Ninth People's Hospital (Shanghai, China) between January 2013 and December 2015 were included in the present analysis. Only nevi present at birth and with a PAS of $\geq 20 \mathrm{~cm}$ were included. For the diagnosis in children, considering that the lesion increases as the body grows, DeDavid's estimating curves were used, via which the diameter of the skin surface was calculated for patients of both sexes in different age groups (9). A total of 98 GCMN cases were identified and specimens were obtained 
during surgical intervention. Age, sex, nevus location, size and clinical appearance were recorded. For comparison, $30 \mathrm{CMN}$ specimens (13 males and 17 females between 2 and 39 years old) were obtained from patients admitted to the Plastic Surgery Department of Shanghai Ninth People's Hospital between January 2015 and December 2015. Patients with neurofibroma associated with melanocytic nevi and acquired nevi were excluded from the present study. All patients or their parents gave their written informed consent for the use of their tissue specimens in this study. The study was approved by the Ethics Committee of Shanghai Ninth People's Hospital (Shanghai, China).

Histochemical staining. The specimens were routinely fixed in $4 \%$ paraformaldehyde and embedded in paraffin wax. Sections $(4 \mu \mathrm{m})$ were obtained and histochemical staining was performed with hematoxylin and eosin as well as Masson trichrome (for collagen). For hematoxylin and eosin staining, the sections were stained for hematoxylin for $7 \mathrm{~min}$, differentiate in $1 \%$ acid alcohol for $10 \mathrm{sec}$, rinse in running water for $30 \mathrm{~min}$, stain with eosin for $1 \mathrm{~min}$. For Masson trichrome staining, the specimens were stained with hematoxylin stain solution for 1 min and acid Fuchsin stain solution for 30-60 sec, differentiated in phosphomolybdic-phosphotungstic acid solution for $6 \mathrm{~min}$, and finally incubated in aniline blue counterstain for $5 \mathrm{~min}$. All the staining procedures were done in room temperature.

Immunohistochemistry (IHC). IHC studies were performed on 4- $\mu \mathrm{m}$ sections of paraformaldehyde-fixed, paraffin-embedded tissue using standard techniques. Antigen retrieval was performed by boiling in citrate buffer $(\mathrm{pH} \mathrm{6.0)}$ ) for $10 \mathrm{~min}$. Primary antibodies against Ki-67 (cat. no. ab15580; 1:200 dilution; Abcam, Cambridge, UK), HMB-45 (cat. no. M063429-2; 1:50 dilution; Dako; Agilent Technologies, Inc., Santa Clara, CA, USA), Melan-A (cat. no. sc20032; Santa Cruz Biotechnology, Inc., Dallas, TX, USA), S100 (cat. no. sc52205; 1:200 dilution; Santa Cruz Biotechnology, Inc.) and Sox10 (cat. no. ab221733; 1:200 dilution; Abcam) were incubated at $4^{\circ} \mathrm{C}$ overnight. Endogenous peroxidase was quenched by incubating the sections for $10 \mathrm{~min}$ with $0.3 \%$ hydrogen peroxide in methanol. Then the sections were incubated with horseradish peroxidaseconjugated goat anti-mouse (cat. no. ab6789) or goat anti-rabbit (cat. no. ab6721; both 1:1,000 dilution; Abcam) secondary antibodies for $1 \mathrm{~h}$ at room temperature. 3-amino-9-ethylcarbazole was the chromogenic substrate. Sections were counterstained with hematoxylin for $2 \mathrm{~min}$ at room temperature. Melanoma tissue and normal skin tissue from Pathology Department of Shanghai Ninth People's Hospital were used for positive and negative controls, respectively. A total of 5 melanoma tissue specimens ( 3 males and 2 females between 58 and 72 years old) were collected between July 2012 and February 2014. A total of normal skin tissue specimens ( 1 males and 4 females between 21 and 46 years old) were collected from January 2013 to May 2014. IHC reactivity was independently scored as positive or negative by MW and QY. The two authors achieved the same results, which is indicative of the ease of reproducibility.

Histopathological evaluation. Digital images of the specimens were captured and analyzed with Image-Pro Plus

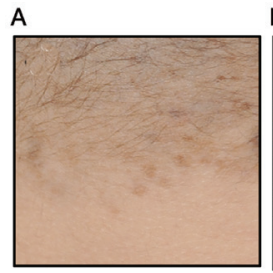

B

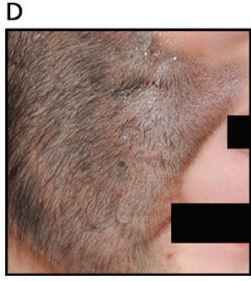

E
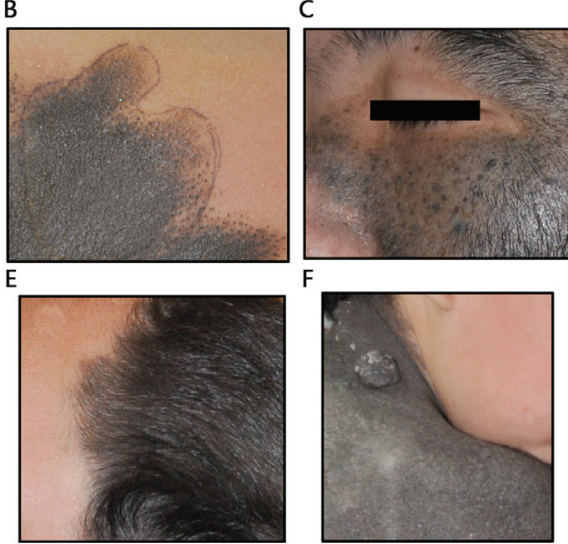

Figure 1. Clinical appearance of giant congenital melanocytic nevi (A) Brown lesion with light hair. (B) Deep black lesion with no hair. The drawn line indicates the area marked for surgical resection. (C) Black lesion with scattered spots. (D) Brown lesion with dense hair. The drawn line indicates the area marked for surgical resection. (E) Deep black lesion with dense hair. (F) Deep black lesion with proliferative nodule (note the poor skin texture of the lesion)

software version 6.0 (Media Cybernetics, Rockville, MD, USA). Histopathological features were evaluated based on standard H\&E-stained sections and Masson trichrome-stained sections. The nevi cell network, depth of invasion, involvement of cutaneous appendages, cytological atypia and architectural features were then determined. The criteria of architectural disorder and the grading of cytological atypia were based on standard criteria mentioned in the literature $(10,11)$. The cell density was measured in lesions with deep or light color, in $1,000 \mu \mathrm{m}$ depth below the epidermal basement membrane, and a square measuring $500 \times 500 \mu \mathrm{m}$ was marked to calculate the cell density. Five visual fields of each sample were randomly selected for calculating, and the evaluation was performed at a magnification of $x 100$. In the specimens from non-surgical treatment (chemical peeling or laser therapy), the depth from the epidermal basement membrane to the dermal treatment plane was measured under a microscope with Image-Pro Plus software.

Statistical analysis. Statistical analysis was performed using SPSS version 11.5 software (SPSS, Inc., Chicago, IL, USA). All of the quantitative values were expressed as the mean \pm standard deviation. Student's t-test was used to determine the significant differences between the two groups. $\mathrm{P}<0.05$ was considered to indicate a statistically significant difference.

\section{Results}

Clinical features. A total of 98 patients diagnosed with GCMN, comprising 57 males (58.2\%) and 41 females (41.8\%), aged from 3 months to 37 years (median age, 6 years), were included. The most common localization of the nevi was the head and neck (44.9\%), followed by the trunk (31.6\%) and extremities (23.5\%). Light black was the most common nevi color (39.8\%), followed by deep black (32.7\%) and brown (27.5\%). Most of the lesions were covered with dense hair (72.4\%); the lesion color was not associated with this feature (Fig. 1). Regarding prior treatment, 10 patients (10.2\%) had 
Table I. Clinical features of patients with giant congenital melanocytic nevi ( $\mathrm{n}=98$ patients).

\begin{tabular}{lc}
\hline Feature & N $(\%)$ \\
\hline Sex & \\
Male & $57(58.2)$ \\
Female & $41(41.8)$ \\
Location of lesion & \\
Head and neck & $44(44.9)$ \\
Trunk & $31(31.6)$ \\
Extremities & $23(23.5)$ \\
Color of lesion & \\
Light black & $39(39.8)$ \\
Deep black & $32(32.7)$ \\
Brown & $27(27.5)$ \\
Hair on lesion & \\
Yes & $71(72.4)$ \\
No & $27(27.6)$ \\
\hline
\end{tabular}

Table II. Histopathological characteristics of light- and deep-color lesions.

\begin{tabular}{lcc}
\hline Item & Light color & Deep color \\
\hline Cell density $^{\mathrm{a}}\left(\mathrm{n} / 250,000 \mu \mathrm{m}^{2}\right)$ & $114.6 \pm 20.9$ & $257.8 \pm 23.9^{\mathrm{b}}$ \\
Depth of cell infiltration & Whole dermis & Whole dermis \\
\hline
\end{tabular}

aAt the plane of $1,000 \mu \mathrm{m}$ below the epidermal basement membrane; ${ }^{\mathrm{b}} \mathrm{P}<0.01$ compared with light-color lesion group.

received partial non-surgical therapy, 7 received laser therapy and 3 had been subjected to chemical peeling. Table I presents the clinical features of the lesions.

IHC characteristics. Nuclear immunoreactivity of dermal nevi cells was noted for $\mathrm{Ki}-67$, but the overall positive rate of dermal nevi cells was low, ranging from 1-9\% (Fig. 2A). All of the lesions exhibited diffuse dermal reactivity to S100 and Melan-A antibodies (Fig. 2B and C). HMB-45 expression was observed in the superficial dermis (Fig. 2D). In addition, all nevi cells exhibited marked and clear nuclear staining for Sox10 (Fig. 2E).

Histological evaluation. All of the GCM had epidermal flattening with loss of the rete ridge pattern. Most of the GCMN samples were composed of intradermal nevi (95.9\%)-nevi cells infiltrating the whole dermis and even into the subcutaneous tissue. Visible pigment granules were identified around superficial nevus cells. The distribution patterns of nevus cells in the dermis were diverse; nevus cells may distribute evenly in dermis (62 out of $98 ; 63.3 \%$ ), or be cord-like between collagen bundles (23 out of 98; 23.5\%); certain lesions presented with nevus cells gathered in nests (14 out of 98; 14.3\%; Fig. 3A-C).

Generally, nevus cells in the superficial portion are rounded-to-polygonal cells with uniform nuclei, i.e., type-A cells.
With increasing depth of the location, cells transform to type-C spindle or fibroblast-like nevomelanocytes, although lesions with only spindle- or fibroblast-like nevus cell distribution were also present in certain cases. Arrector pili muscles, hair follicles and sebaceous gland were occasionally involved (Fig. 3D-G). Nevus cells were also observed around capillaries. Cell atypia is commonly identified in such lesions, but mitosis is rarely present.

When texture was taken into account, it was noted that the more dermal infiltration and collagen destruction occurred, the poorer the skin texture was. Lesions of different color were compared, and the cell density among different lesions was measured at the same depth $(1,000 \mu \mathrm{m}$ below the epidermal basement membrane) under a microscope. The results demonstrated that deep-colored lesions had a higher nevus cell density compared with light-colored lesions $(257.8 \pm 23.9$ vs. $114.6 \pm 20.9$ cells per $250,000 \mu \mathrm{m}^{2}$; P<0.01; Fig. 4 A-C). As to the depth of nevus cell infiltration, it was observed that in all of the GCMN lesions, the nevus cells defused deep into the whole dermis and even infiltrated into subdermal adipose tissue (Table II). Of note, most of the GCMN cases $(95.9 \%)$ had a sub-epidermal non-involvement zone, which is quite similar to a Grenze zone, and a normal collagen band was observed by Masson staining (Fig. 2F). The width of this sub-epidermal non-involvement zone was measured in all specimens, and the average value was $292.8 \pm 74.4 \mu \mathrm{m}$. Small CMN specimens featured nevus cells involving all of the superficial dermis and partially the epidermis in a junctional nevi pattern (Fig. 2G).

Non-surgical therapy. In the present study, 10 patients were subjected to non-surgical therapy. The histopathological changes induced by these therapies were evaluated. Laser treatment specimens indicated that laser treatment only removed melanin in the superficial dermal plane (Fig. 4E); hence, slight collagen hyperplasia remained and the intradermal nevus cells were not involved. Indeed, the whole skin structure appeared relatively unchanged.

In chemical peeling specimens, the upper third of the dermal nevus cells and melanin were cleaned and replaced by thick collagen (Fig. 4D); the depth of the chemical peeling treatment was measured in 3 specimens and the average depth of this treatment was $1,031.2 \pm 158.9 \mu \mathrm{m}$. High counts of inflammatory cells infiltrated into the remaining nevi cells in the deep dermis, and remaining nevi cells exhibited no obvious morphological changes. The gross appearance after chemical peeling therapy indicated visible improvement of the pigment appearance, albeit with scarring.

\section{Discussion}

The current knowledge regarding GCMN is poor, and previous studies mainly focus on clinical and histopathological characteristics of CMN (12-15). Although GCMN is a sub-group of CMN, these nevi have a different mutation spectrum and a higher malignant transformation rate. Chung et al (16) reviewed giant nevi, describing the epidemiological characteristics and treatment of GCMN. Furthermore, Arneja and Gosain (17) reported on their experience treating GCMN. However, studies on the histopathological features of GCMN are lacking and are urgently required. 

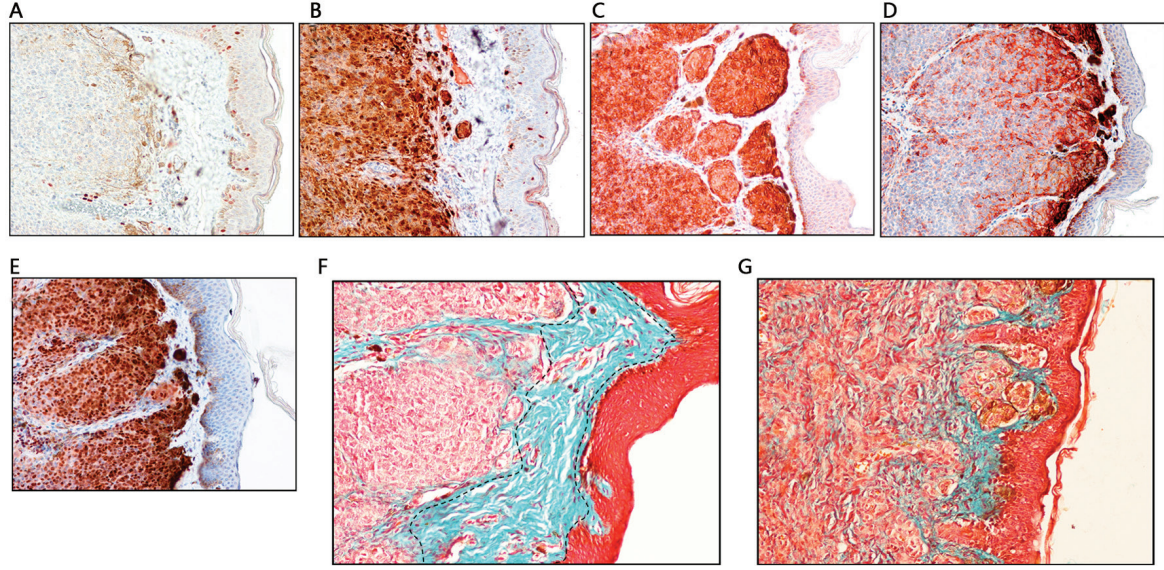

Figure 2. Immunohistochemical features of giant CMN. (A) The Ki67-positive rate is low in dermal nevus cells (magnification, x100). (B) All dermal nevus cells exhibited S100 reactivity. (C) Melan-A positive staining in dermal nevus cells (magnification, x200). (D) Only the upper portion of nevus cells have HMB45 reactivity (magnification, x100). (E) All nevi cells exhibited an intense and clear nuclear SRY-related HMG-box 10 positive staining (magnification, x100). (F) Masson trichrome staining displaying a band-shaped nevus cell-poor zone with normal collagen in this zone (broken line; magnification, x200). (G) CMN lesion demonstrates the involvement of the basal layer and absence of a nevus cell-poor zone (magnification, x200). CMN, congenital melanocytic nevi.

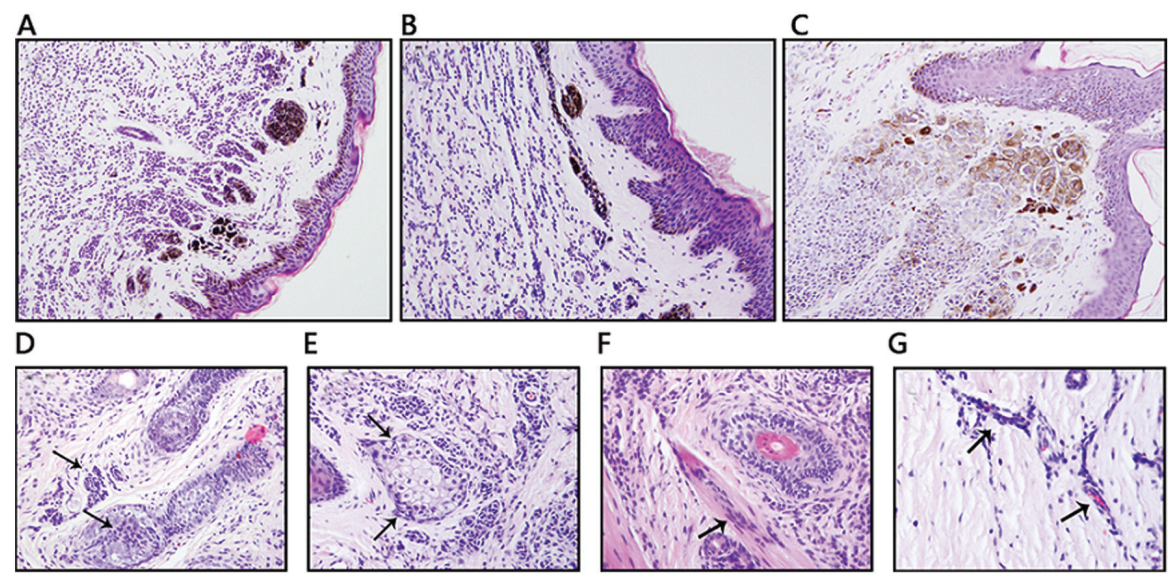

Figure 3. Distribution patterns of nevus cells and skin adnexal involvement. (A) Even distribution of nevus cells in the dermis. (B) Cord-like distribution of nevus cells between collagen bundles. (C) Nevus cells gathered in nests (magnification, x100). (D) Hair follicle involvement in dermis. (E) Sebaceous gland involvement. (F) Nevus cells infiltrated into the arrector pili muscle. (G) Nevus infiltration around capillary vessel (arrows indicate nevus cells surrounding capillary vessels; magnification, $\mathrm{x} 200)$.
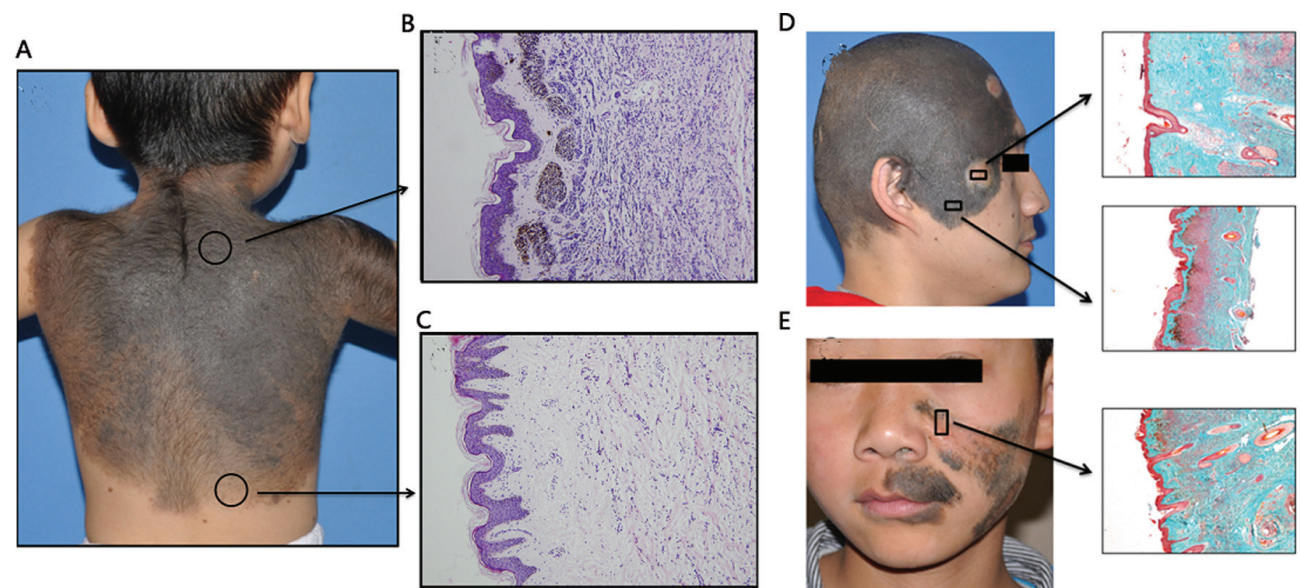

Figure 4. (A) Large giant congenital melanocytic nevi lesion on the back of a 3-year-old boy. (B) Deep-colored lesion exhibiting high-density nevus cell infiltration and extensive melanin deposition in the upper dermis (magnification, x100). (C) Light-colored lesion exhibiting low-density nevus cell infiltration and little melanin deposition. (D) Sample from chemical peeling area indicates that the upper portion of the nevus cells and deposited melanin were removed and replaced with a layer of dense scar tissue; the average depth of treatment was $1,031.2 \pm 158.9 \mu \mathrm{m}$. Deep subcutaneous nevus cells were left behind with no morphological changes were observed. (E) Sample form junctional zone after laser therapy. The lower part of the section is the area subjected to laser treatment; the deposited melanin was removed and the subcutaneous nevus cells were left behind with a layer of superficial scar tissue overlaying them. 
When considering the anatomic distribution of nevi, Mark et al (18) reported that CMN was distributed as follows: $38 \%$ on the trunk, $38 \%$ on the legs and arms, $14 \%$ on the head and neck, and $10 \%$ on the feet and hands. Sahin et al (19) investigated only medium-sized CMN, and indicated that the most common localization was on the head and neck, followed by anterior and posterior trunk. In the present cohort of GCMN cases, the most common localization was on the head and neck (44.9\%), followed by trunk (31.6\%) and extremities (23.5\%).

The distribution pattern in the present study was similar to that reported by Sahin et al (19), which suggests large-sized nevi may be different from small CMN regarding their distribution. Mark et al (18) indicated that nearly all CMN are brown, but according to the present results, light and deep black were the most common color of GCMN. This color difference between studies may be explained by more dense nevi cells infiltration and melanin synthesis in GCMN. The color depth is correlated with nevus cell infiltration, and thus, the deeper the color, the more nevus cell infiltration and superficial melanin deposition are present. Skin texture is also associated with the infiltration of nevus cells; infiltrating cells destroy dermal collagen, and thus, the skin texture is affected. It may therefore be recommended that nevi cell infiltration is estimated by noting the texture and color of the nevus.

In the present study, the IHC characteristics of GCMN were determined by evaluation of Ki67, Melan-A, S-100, HMB-45 and SOX-10 immunopositivity in GCMN lesions. The nuclear proliferation marker Ki67 assumes an important role in the distinction between melanocytic lesions; benign nevi have proliferation indices of $<5 \%$, while the average index of melanomas is $27 \%$ (range, 5-50\%) $(20,21)$. The present study indicated that the overall positive rate was low (ranging from 1-6\%), which is quite close to the rate reported in CMN by Lebe et al (22). The result implied that the size of the lesion is not associated with the proliferative activity. The overall low $\mathrm{Ki}-67$ labeling observed in the present study is consistent with the benign nature of GCMN. HMB45 was discovered in 1986 from an extract of melanoma, and the antibody detecting the cytoplasmic pre-melanosomal glycoprotein was named gp100 (23). In CMN, HMB45 staining is usually restricted to the junction; in the present cohort of GCMN cases, even upper-layer nevus cells exhibited HMB45 staining, which proves that nevus cells in the superficial layer of GCMN are more active than those of CMN. This may explain for the higher malignant transformation rate of GMCN.

SOX10 is a key molecule in the embryonic development of melanocytes. Haploinsufficiency of SOX10 causes aganglionosis of the colon and pigmentation defects. Extensive research has revealed that SOX10 has an important role in GCMN and melanoma $(24,25)$. The present study demonstrated that nevus cells in GCMN exhibited marked nuclear staining for SOX10. However, the underlying molecular mechanisms via which SOX10 exerts its effects remain elusive.

Previous studies summarized the pathological characteristics of CMN and concluded that nevus cells extend between collagen bundles of the reticular dermis as single cells or cords of cells (26). The present study indicated that in certain cases of dermal GCMN, nevus cell infiltration is relatively dense and the phenomenon of single or cords of cells extending between collagen may not appear.
In the present study, 95.9\% of the GCMN featured a sub-epidermal non-involvement zone, which is different in small CMN, while numerous CMN cases had a junctional and compound pattern. This is a noteworthy phenomenon, which reflects the derivation of the nevus. Mutation of melanoblasts during migration in the embryonic stage leads to development into a nevus, and mutations at different time-points cause different clinical and histopathological characteristics. Earlier mutations in migration stages result in a larger nevus size with a deeper infiltration, since a certain distance is present between the proliferated nevus cells and epidermis, so that a nevus cell-poor zone is formed under the epidermis. If a mutation occurs in a late stage, the resulting nevus is smaller in size and proliferating nevus cells are close to the epidermis or even involved in the basal layer of the epidermis.

Non-surgical techniques, including chemical peeling and laser therapy, are occasionally used to treat GCMN. Although it has been suggested that laser therapy is effective in removing small melanocytic nevi (27), the present study suggests that it only attacks the superficial, pigment-bearing portion of the nevus and sub-cutaneous nevus cells are left behind and covered with a layer of superficial scar tissue. Indeed, the remaining nevus cells in the deep dermis still possess a malignant transformation capability, as melanoma formation in GCMN was reported after dermabrasion $(28,29)$. With this regard, caution is therefore warranted when selecting a non-surgical therapy for GCMN.

The current knowledge on GCMN is limited, and the mechanisms underlying its genesis and development have remained to be fully elucidated. In the present study, a large cohort of GCMN cases demonstrated diverse clinical appearances and histopathological characteristics. Different distribution patterns of nevus cells were identified among GCMN cases, which were also quite different from CMN. The proliferation rate of nevus cells was similar, and HMB-45 staining was more intense in the upper portion of nevi. A sub-epidermal non-involvement zone was identified in most of the GCMN, which is indicative of their different origin from that of $\mathrm{CMN}$, and is in accordance with the differences in mutation spectrum between GCMN and small CMN.

In summary, GCMN have unique morphological and IHC features compared with small CMN or AMN. Due to their higher malignant transformation rate and poorer cosmetic appearance, GCMN requires a different and urgent therapeutic strategy. The present study performed an in-depth summary of the histopathological features of GCMN, and provides a deeper understanding of this disease, which will provide a foundation for exploring novel treatment options.

\section{Acknowledgements}

The authors would like to thank Dr Hainan Zhu (Department of Plastic and Reconstructive Surgery, Shanghai Ninth People's Hospital, Shanghai Jiao Tong University, School of Medicine, Shanghai, China) for data collection and insightful discussion.

\section{Funding}

The present study was funded by the Shanghai Pujiang Talents Program [grant no. PJ(2015)0002993] and National Natural Science Foundation of China (grant no. 81871595). 


\section{Availability of data and materials}

All data generated or analyzed during this study are included in this published article.

\section{Authors' contributions}

FX, QL and QY designed the study. BG and LS collected the clinical data. QY, MW and LS performed the experiments and analyzed the data. MW and QY wrote the paper. All authors read and approved the final manuscript.

\section{Ethics approval and consent to participate}

All patients or their parents gave their written informed consent for the use of their tissue specimens in this study. The study was approved by the Ethics Committee of Shanghai Ninth People's Hospital (Shanghai, China).

\section{Patient consent for publication}

All patients or their parents gave their written informed consent for the use of their tissue specimens and images in this study.

\section{Competing interests}

The authors declare no competing interests.

\section{References}

1. Kopf AW, Bart RS and Hennessey P: Congenital nevocytic nevi and malignant melanomas. J Am Acad Dermatol 1: 123-130, 1979.

2. Hashmi GS, Ahmed SS and Khan S: Congenital giant melanocytic nevi. Rare Tumors 1: e9, 2009.

3. Lyon VB: Congenital melanocytic nevi. Pediatr Clin North Am 57: 1155-1176, 2010.

4. Arad E and Zuker RM: The shifting paradigm in the management of giant congenital melanocytic nevi: Review and clincal applications. Plast Reconstr Surg 133: 367-376, 2014.

5. Watt AJ, Kotsis SV and Chung KC: Risk of melanoma arising in large congenital melanocytic nevi: A systematic review. Plast Reconstr Surg 113: 1968-1974, 2004.

6. Krengel S, Hauschild A and Schafer T: Melanoma risk in congenital melanocytic naevi: A systematic review. Br J Dermatol 155: 1-8, 2006.

7. Bett BJ: Large or multiple congenital melanocytic nevi: Occurrence of cutaneous melanoma in 1008 persons. J Am Acad Dermatol 52: 793-797, 2005.

8. Hale EK, Stein J, Ben-Porat L, Panageas KS, Eichenbaum MS, Marghoob AA, Osman I, Kopf AW and Polsky D: Association of melanoma and neurocutaneous melanocytosis with large congenital melanocytic naevi-results from the NYU-LCMN registry. Br J Dermatol 152: 512-517, 2005.

9. Charbel C, Fontaine RH, Malouf GG, Picard A, Kadlub N, El-Murr N, How-Kit A, Su X, Coulomb-L'Hermine A, Tost J, et al: NRAS mutation is the sole recurrent somatic mutation in large congenital melanocytic nevi. J Invest Dermatol 134: 1067-1074, 2014

10. Murphy GF and Mihm MC: Recognition and evaluation of cytological dysplasia in acquired melanocytic nevi. Hum Pathol 30: 506-512, 1999.
11. Weinstock MA, Barnhill RL, Rhodes AR and Brodsky GL: Reliability of the histopathologic diagnosis of melanocytic dysplasia the dysplastic nevus panel. Arch Dermatol 133: 953-958, 1997.

12. DeDavid M, Orlow SJ, Provost N, Marghoob AA, Rao BK, Wasti Q, Huang CL, Kopf AW and Bart RS: Neurocutaneous melanosis: Clinical features of large congenital melanocytic nevi in patients with manifest central nervous system melanosis. J Am Acad Dermatol 35: 529-538, 1996.

13. Price HN and Schaffer JV: Congenital melanocytic nevi-when to worry and how to treat: Facts and controversies. Clin Dermatol 28: 293-302, 2010.

14. Alikhan A, Ibrahimi OA and Eisen DB: Congenital melanocytic nevi: Where are we now? Part i. clinical presentation, epidemiology, pathogenesis, histology, malignant transformation, and neurocutaneous melanosis. J Am Acad Dermatol 67: 495 e1-e17, 2012.

15. Gallus S and Naldi J; Oncology Study Group of the Italian Group for Epidemiologic Research in Dermatology: Distribution of congenital melanocytic naevi and congenital naevus-like naevi in a survey of 3406 Italian school children. Br J Dermatol 159: 433-438, 2008.

16. Chung C, Forte AJ, Narayan D and Persing J: Giant nevi: A review. J Craniofac Surg 17: 1210-1215, 2006.

17. Arneja JS and Gosain AK: Giant congenital melanocytic nevi. Plast Reconstr Surg 124(Suppl 1): e1-e13, 2009.

18. Mark GJ, Mihm MC, Liteplo MG, Reed RJ and Clark WH: Congenital melanocytic nevi of the small and garment type. Clinical, histologic, and ultrastructural studies. Hum Pathol 4: 395-418, 1973.

19. Sahin S, Levin L, Kopf AW, Rao BK, Triola M, Koenig K, Huang C and Bart R: Risk of melanoma in medium-sized congenital melanocytic nevi: A follow-up study. J Am Acad Dermatol 39: 428-433, 1998.

20. Nasr MR and El-Zammar O: Comparison of pHH3, Ki-67, and survivin immunoreactivity in benign and malignant melanocytic lesions. Am J Dermatopathol 30: 117-122, 2008.

21. Ohsie SJ, Sarantopoulos GP, Cochran AJ and Binder SW: Immunohistochemical characteristics of melanoma. J Cutan Pathol 35: 433-444, 2008.

22. Lebe B, Pabuccuoglu U and Ozer E: The significance of Ki-67 proliferative index and cyclin D1 expression of dysplastic nevi in the biologic spectrum of melanocytic lesions. Appl Immunohistochem Mol Morphol 15: 160-164, 2007.

23. Shakhova O, Zingg D, Schaefer SM, Hari L, Civenni G, Blunschi J, Claudinot S, Okoniewski M, Beermann F, Mihic-Probst D, et al: Sox 10 promotes the formation and maintenance of giant congenital naevi and melanoma. Nat Cell Biol 14: 882-890, 2012.

24. Rothberg BE, Moeder CB, Kluger H, Halaban R, Elder DE, Murphy GF, Lazar A, Prieto V, Duncan LM and Rimm DL: Nuclear to non-nuclear Pmel17/gp100 expression (HMB45 staining) as a discriminator between benign and malignant melanocytic lesions. Mod Pathol 21: 1121-1129, 2008.

25. Bakos RM, Maier T, Besch R, Mestel DS, Ruzicka T, Sturm RA and Berking C: Nestin and SOX9 and SOX10 transcription factors are coexpressed in melanoma. Exp Dermatol 19: e89-e94, 2010.

26. Tannous ZS, Mihm MC Jr, Sober AJ and Duncan LM: Congenital melanocytic nevi: Clinical and histopathologic features, risk of melanoma, and clinical management. J Am Acad Dermatol 52: 197-203, 2005.

27. Lee SE, Choi JY, Hong KT and Lee KR: Treatment of acquired and small congenital melanocytic nevi with combined Er: YAG laser and long-pulsed alexandrite laser in Asian skin. Dermatol Surg 41: 473-480, 2015.

28. Zutt M, Kretschmer L, Emmert S, Haenssle H, Neumann C and Bertsch HP: Multicentric malignant melanoma in a giant melanocytic congenital nevus 20 years after dermabrasion in adulthood. Dermatol Surg 29: 99-101, 2003.

29. Kishi K, Matsuda N, Kubota Y, Katsube KI, Imanishi N and Nakajima T: Rapid, severe repigmentation of congenital melanocytic naevi after curettage and dermabrasion: Histological features. Br J Dermatol 156: 1251-1257, 2007. 